\title{
Preface to Special Section on Climate Models at the Max Planck Institute for Meteorology
}

Understanding the internal and externally forced dynamics of the climate system has been the major research goal of the Max Planck Institute for Meteorology (MPI-M) since its foundation. Numerical models are considered as essential to test our knowledge on the functioning of the climate system as a whole or in parts, and to explore consequences of changed boundary conditions. Therefore, a palette of numerical models for atmosphere, ocean, and land has been developed and used in the global or regional context. This special issue now presents the most recent set of global models developed at the MPI-M, used here to study various aspects in the earth system. The development of these models often includes contributions from guest scientists or other institutions, which we want to acknowledge explicitly. Sufficiently tested models are distributed to the community (see http://www.mpimet.mpg.de/en/wissenschaft/modelle/model-distribution. html).

The atmospheric general circulation model ECHAM5 is the latest model cycle of the ECHAM series. ECHAM5 is used in two major configurations: the tropospheric model resolving the atmosphere up to $10 \mathrm{hPa}$ and the middle atmosphere model, referred to as MAECHAM5, with a top layer centered at $0.01 \mathrm{hPa}$. The Hamburg Model of the Neutral and Ionized Atmosphere (HAMMONIA), which extends from the surface to the lower thermosphere, is a coupled chemistry atmosphere model based on MAECHAM5 and the chemistry of the Model for Ozone and Related Tracers, version 3 (MOZART3), chemistry transport model. The ECHAM5-HAM model has been developed to investigate the role of tropospheric aerosols in the climate system. The most recent ocean general circulation model, the MPI Ocean Model (MPI-OM), is coupled to EHCAM5 using the Ocean Atmosphere Sea Ice Soil (OASIS3) coupler of the European Parameter Elevation Regressions on Independent Slopes Model (PRISM) model-coupling framework. This coupled system has been used for the German Intergovernmental Panel on Climate Change (IPCC) fourth assessment report (AR4) simulations and forms the physical core of more complex earth system models (ESMs).

Two particular ESMs have been developed and used after submission of this special issue: the carbon cycle ESM based on ECHAM5/MPI-OM and additional submodels for the land vegetation and the marine biogeochemistry, and the aerosol ESM linking marine dimethyl sulfide (DMS) emissions and sulfate aerosol formation, oceanic dust deposition, and plankton production. Future developments will aim at the scientific and technical refinement of the emerging ESMs within the Community Earth System Models network that includes the Max Planck Institutes for Meteorology, Chemistry, and Biogeochemistry, and universities in Europe and Germany. Major challenges will be the modeling of substance cycles and their role in various compartments of the earth system and in climate dynamics, and eventually the coupling of the social system currently treated as an external factor.

The articles of the special section discuss the sensitivity of basic tropospheric metrics to horizontal and vertical resolution (Roeckner et al.), radiative fluxes (Wild and Roeckner), the hydrological cycle (Hagemann et al.), and snow cover and albedo (Roesch and Roeckner). Emission-induced nonlinearities in the global aerosol system are presented by Stier et al. The El Niño response of the northern polar vortex is investigated in ensemble simulations (Manzini et al.), and the quasi-biennial oscillation (QBO) simulated in a high vertical resolution integration is shown in Giorgetta et al. Schmidt et al. analyze the sensitivity of the 
mesopause region to the 11-yr solar cycle and $\mathrm{CO}_{2}$ doubling, and Brasseur et al. (2006) investigate the impact of climate change on the future chemical composition of the global troposphere. Ocean circulation and tropical variability in the coupled model ECHAM5/MPI-OM, which has been used for IPCC AR4 simulations, are presented by Jungclaus et al., and effects of coupling the light penetration to plankton amounts in the ocean surface layer are described in Wetzel et al.

Marco A. Giorgetta, Guy P. Brasseur,* Erich Roeckner, and Jochem Marotzke

* At NCAR since January 2006 